\title{
Substrate Recognition and Catalysis by Flap Endonucleases and Related Enzymes
}

${ }^{1}$ Christopher G. Tomlinson, ${ }^{1}$ John M. Atack, ${ }^{2}$ Brian Chapados, ${ }^{2,3}$ John A. Tainer, ${ }^{1,4}$ Jane A. Grasby

${ }^{1}$ Centre for Chemical Biology, Department of Chemistry, Krebs Institute, University of Sheffield, Sheffield, S3 7HF, UK.

${ }^{2}$ Skaggs Institute for Chemical Biology, The Scripps Research Institute, Department of Molecular Biology - MB4, 10550 North Torrey Pines Road, La Jolla, CA 92037, USA

${ }^{3}$ Life Sciences Division, Department of Molecular Biology, Lawrence Berkeley National Laboratory, Berkeley, CA 94720, USA

${ }^{4}$ Corresponding Author j.a.grasby @ sheffield.ac.uk 


\section{Abstract}

Flap endonucleases (FENs) and related FEN-like enzymes (EXO-1, GEN-1 and XPG) are a family of divalent metal ion dependent nucleases that catalyse structure-specific hydrolyses of DNA duplex containing nucleic acid structures during DNA replication, repair and recombination. In the case of FENs, the ability to catalyse reactions on a variety of substrates has been rationalised as a result of combined functional and structural studies. Analyses of FENs also exemplify controversies regarding the two metal ion mechanism. However, kinetic studies of T5FEN reveal that a two metal ion like mechanism for chemical catalysis is plausible. Consideration of the metallobiochemistry and the positioning of substrate in metal-free structures has led to the proposal that the duplex termini of substrates are unpaired in the catalytically active form and that FENs and related enzymes may recognise breathing duplex termini within more complex structures. An outstanding issue in FEN catalysis is the role played by the intermediate (I) domain arch or clamp. It has been proposed that FENs thread the 5'-portion of their substrates through this arch, which is wide enough to accommodate single but not double stranded DNA. However, FENs exhibit gap endonuclease activity acting upon substrates that have a region of 5'-duplex. Moreover, the action of other FEN family members such as GEN-1, proposed to target Holliday Junctions without termini, appears incompatible with a threading mechanism. An alterative is that the I domain is used as a clamp. A future challenge is to clarify the role of this domain in FENs and related enzymes.

Key words: FEN-1, XPG, GEN-1, EXO-1, metal ions, nuclease, DNA repair 
Abbreviations used: FEN flap endonuclease; T5FEN bacteriophage T5 flap endonuclease (in early literature referred to as T5 5'-3'-exonuclease and T5 5'-nuclease) AfFEN Archaeoglobus fulgidus flap endonuclease; MjFEN Methanococcus jannaschii flap endonuclease; T4FEN T4 flap endonuclease (referred to also as T4 RNase H); hFEN-1 human flap endonuclease; EXO-1 exonuclease-1; XPG xeroderma pigmentosum complementation group G; GEN-1 XPG-like gap endonuclease (putative human Holliday junction resolvase); nt nucleotide; XPF xeroderma pigmentosum complementation group $\mathrm{F}$ 


\section{Introduction}

Flap endonculeases (FENs) are divalent metal ion dependent, structure-specific, nucleic acid hydrolysing enzymes that play critical roles in DNA replication and repair [1, 2]. In replication and certain types of DNA repair they are responsible for removal of the primers present as 5'-flaps. In vitro FENs possess multiple structure specific DNA hydrolysing activities catalysing both the endonucleolytic reactions of 5'-bifurcated substrates and the 5'-exonucleolytic reactions of blunt ended DNAs (Figure 1a) [3-5]. All FEN activities proceed from the same active site and have an absolute requirement for divalent metal ion cofactors. In higher organisms other FEN-like proteins have been identified on the basis of sequence alignments and are involved in DNA repair and recombination. In humans these hFEN-1 paralogues are EXO-1, GEN-1 and XPG [6-10]. All of these enzymes can carry out the FEN-type flap endonucleolytic reactions on 5'-flap structures in vitro, but the in vivo substrates of GEN-1 and XPG are proposed to be Holliday junctions and DNA repair bubbles respectively (Figure 1a) [6, 9, 10].

\section{FEN structures, FEN-like protein organisation and FEN-substrate interactions}

Since their initial discovery over two decades ago significant advances have been made towards understanding the molecular basis of FEN action. Structures of FENs from multiple domains of life have highlighted that a common protein structure has been maintained to sustain FEN activity from bacteriophages through archaea to humans [1119]. The protein has a two domain saddle like structure, formed from the $\mathrm{N}$ and $\mathrm{C}$ terminal parts of the protein, that are joined by an intermediate (I) domain (Figure 1b). In archaea as well as higher eukaryotes C-terminal extensions act as interaction sites for other proteins $[14,16,18]$. EXO-1, GEN-1 and XPG appear to contain the same overall domain organisation [6-10]. In structures of bacteriophage T5 FEN (T5FEN) and Archaeoglobus fulgidus FEN (AfFEN) the I domain is observed to form a helical arch [13, 18], but appears more clamp-like in the structure of Pyrococcus furiosis FEN [14] and various states of order/disorder have been observed in other structures (Figure 1c and d). An initial puzzle was the ability of FENs to act on multiple DNA structures. Through a combination of protein mutagenesis, biophysical and kinetic experiments, studies using altered DNA substrates and finally structural studies of DNA protein complexes, an 
understanding of the mode of interaction of FEN substrates with the enzyme has emerged (Figure 1c and d) [18-22]. A characteristic of FEN reactions is that the major site of reaction occurs one nucleotide (nt) into the double stranded region. The duplex downstream of the main site of reaction is bound in the C-terminal domain by a modified form of the non-specific duplex DNA interaction motif, the helix-three-turn-helix, a variant of the helix-hairpin-helix motif first discovered in the endonuclease III structure [23]. Duplex or single stranded DNA upstream of the major site of FEN action is bound by the N-terminal domain and is bent at a $90^{\circ}$ angle with respect to the downstream strand $[18,19]$.

Higher organism FENs have enhanced specificity for reaction one nt into the duplex region in comparison to their lower organism counterparts, providing the substrate has an upstream duplex with a single nt 3'-flap [24]. Substrates with 5'-flaps and a 3'-extra nt (so-called double flap substrates) are processed with enhanced reaction rates by hFEN1[5]. A binding site for the 3'-extra nt exits in higher organism FENs, formed from helices not present in bacteriophage FENs [4, 19, 25] (Figure 1c). Forming this interaction presumably restricts the substrate to a unique interaction mode with the protein that gives rise to enhanced specificity [18]. The product of higher organism FEN action is therefore suitable for immediate ligation by DNA ligase. With the exception of this 3'-extra nt, no other contacts are made to this strand of the substrate, but substantive interactions are observed to the opposite (template) strand of the upstream DNA. The hFEN paralogues hEXO-1, hXPG, and hGEN-1 appear to lack the residues associated with the 3'-extra nt binding site, based upon comparisons to the DNA-bound archaeal FEN structure [18], but similar modes of interaction with downstream and upstream DNA appear likely.

\section{FEN active site and metallobiochemistry}

Typical of most metallonucleases, FENs have a carboxylate rich active site that ligands essential divalent metal ion cofactors. This active site is located at the base of the helical arch. However, unlike most other nucleases that contain three or four carboxylates, seven are positionally conserved in all FEN structures and an eighth is conserved in phage and 
bacteria (Figure 2). The positioning of metal ions in FEN structures has provoked much debate. In the initial structures of FENs from bacteriophage T4 and T5 two metal ions where observed within the active sites with a separation of $6.3 \AA$ and $8 \AA$ respectively, too far apart for both ions to contact the same phosphate $[12,13]$. Yet the most common, albeit controversial, mechanism proposed for metallonucleases requires a $<4 \AA$ A separation for ions to act as Lewis acid catalysts and to serve as a source of generating hydroxide ions [26]. Thus several alternative mechanisms have been proposed for FENs [11, 12, 14, 19, 27-29]. Later structures of higher organism FENs from archaea and humans revealed $5 \AA$ and $3.4 \AA$ separation between ions respectively, and notably the site occupied by the metal ion 2 in phage structures contains the $\mathrm{N}$-terminus of these proteins $[15,16]$. One possibility it that metal 2 in phage and bacterial FENs plays an equivalent, but as yet unknown, role to the $\mathrm{N}$-terminus in higher organisms.

A functional study of T5FEN revealed that the overall reaction catalysed by this enzyme $\left(k_{\mathrm{cat}} / K_{\mathrm{M}}\right)$ had a third order dependence on magnesium ion concentration, implying a requirement for at least three metal ions [30]. Maximal multiple and single turnover rates of the reaction required the presence of at least two ions with differing affinities. The magnitude of the Michaelis constant was also observed to be metal dependent and was minimised at higher divalent metal ion concentrations, with $1 / K_{\mathrm{M}}$ displaying a second order dependence on metal ion concentration at lower magnesium ion concentrations. Together this suggests that a two metal ion like mechanism for the FEN reaction is plausible if a third ion is bound close to metal 1 as in the human FEN structure (Figure $2 \mathrm{c}$ ), but that overall the metallobiochemistry is more complex. In line with a requirement for two catalytic ions, non-catalytically active calcium ions inhibit the reaction catalysed by T5FEN competitively with viable cofactor and with a $1 /[\mathrm{Ca}]^{2}$ dependence at higher concentrations of calcium ions. In contrast to the unmodified protein, the overall reaction $\left(k_{\text {cat }} / K_{\mathrm{M}}\right)$ of a mutant of T5FEN where two of the carboxylates that are observed to ligand metal 2 are neutralised is only two metal ion dependent. Evidence for an inhibitory species with two calcium ions bound is also observed with this mutant (Tomlinson and Grasby, unpublished observations). 


\section{FEN unpairs its substrate}

The only structure of a FEN in complex with a whole bifurcated substrate is that of T4FEN (referred to as T4 RNase $\mathrm{H}$ ) in complex with a pseudo-Y DNA [19]. An intriguing feature of this structure is that the major site of FEN reaction one nt into the duplex region of substrates is within the downstream duplex bound parallel to the active site (Figure 3a). In this complex, solved in the absence of metal cofactors, the substrate avoids contact with the active site, presumably as a consequence of carboxylate repulsion. The scissile bond is positioned $7 \AA$ from metal 1 and further from metal 2 observed in a substrate-free structure of the same FEN [19]. However, it is difficult to envisage moving the substrate in its duplex form into the active site. Based upon the metal dependence of substrate equilibria and the structural observations, we suggested that an unpaired form of the substrate would allow the scissile bond to make intimate contact with the metal ion in the active site (Figure 3b) [30]. Thus a feature of FEN substrate interactions may be to recognise and capture a transiently unpaired breathing nucleic acid substrate within the context of a complex DNA structure. We propose that this property is likely shared with XPG, EXO-1 and GEN-1.

\section{How do FENs and FEN-like proteins interact with the 5'-portion of their substrates?}

Another feature of FENs that has attracted much debate is their mode of interaction with the 5'-portion of their substrates (Figure 1d). At physiological $\mathrm{pH}$ FEN substrates with 5'-flaps interact with FEN protein with low $\mathrm{nM}$ dissociation constants in the absence of cofactors, whereas substrates lacking 5'-flaps interact much more weakly [19, 31]. In its structured form the FEN helical arch formed from the I domain is wide enough to accommodate single but not double stranded DNA [13]. This provides a pleasing rationalisation of the much greater affinity of 5'-flap containing substrates, and it was proposed that single stranded DNA flaps were threaded through the arch $[13,32]$. More recently in the structure of T4FEN complexed with pseudo-Y DNA, the 5'-flap potion of the substrate does indeed appear to go though the arch, although a portion of the I domain was disordered in this structure (Figure 1d) [19]. Superposition of the T4 complex with FENs with structured helical arches leads to passage of the 5'-single strand through the 
arch [19]. However, FENs also accommodate substrates where the 5'-portion of the molecule contains a region of duplex and this gap endonuclease activity proceeds with only a 10-fold reduced catalytic efficiency for hFEN-1 [5]. Assuming threading is a prerequisite for reaction then it is possible that duplex DNA could be passed through a disordered arch or that FENs have the capacity to resolve short regions of secondary structure and pass them though the arch as though they are single stranded. Alternatively, substrates with a gapped region could be processed in a different way that does not require passage through the I domain. This could involve using the I domain as a clamp as was suggested on the basis of archaeal FEN structures [14, 18].

As yet there is no published structural information on EXO-1 and GEN-1 and XPG, but EXO-1 and GEN-1 have predicted similar sized I domains to FENs. In contrast the I region of XPG is 700+ amino acids and has been termed the R-domain as a proposed recognition element for XPG partners [33]. However, some conserved higher organism FEN-1 I domain residues are sequence conserved in all related enzymes (Grasby, unpublished observation). As hEXO-1, a recombination exonuclease, displays FEN type exonucleolytic and endonucleolytic activities [9], the action of this enzyme is likely to be similar to FENs. However, the proposed biological substrates of GEN-1 and XPG are Holliday junctions and repair bubbles respectively, neither of which have a free 5'terminus available in vivo [7, 10]. Reconciling the proposed activities of these proteins with a requirement to thread the 5'-portion of substrates through a helical arch is difficult. However, in nucleotide excision repair XPG acts in concert with XPF to excise damaged DNA, and it has recently been demonstrated that the first cut is made by XPF [34]. The action of XPF would reveal a 5'-flap for a FEN type XPG endonucleolytic activity that could potentially involve arch threading. It is possible that GENs could have a recombination function that deals with an alternative recombination intermediate that presents a 5'-terminus even if this portion of the molecule resembles a FEN gap endonucleolytic substrate [6]. Another possibility is that GEN-1 and XPG (and even EXO-1 and FENs) utilise the arch feature as a clamp or in a different way that does not involve passing DNA through it. Further studies are therefore required to clarify the 
functional mode of interaction of 5'-portions of substrates with the I arch/clamp domains of FENs and related enzymes.

\section{Acknowledgements}

We thank Prof. Jon Sayers and members of the Centre for Chemical Biology (Sheffield) and the Structural Cell Biology of DNA Repair (Scripps) for insightful discussions.

\section{Funding}

Our work is supported by grants from the BBSRC (JAG) and NIH (JAT).

\section{References}

1 Liu, Y., Kao, H. I. and Bambara, R. A. (2004) Flap endonuclease 1: a central component of DNA metabolism. Annu. Rev. Biochem. 73, 589-615

2 Lieber, M. R. (1997) in Bioessays, vol. 19, pp. 233-240

3 Wu, X. T., Li, J., Li, X. Y., Hsieh, C. L., Burgers, P. M. J. and Lieber, M. R. (1996) Processing of branched DNA intermediates by a complex of human FEN-1 and PCNA. Nucl. Acids Res. 24, 2036-2043

4 Williams, R., Sengerová, B., Osborne, S., Syson, K., Ault, S., Kilgour, A., Chapados, B. R., Tainer, J. A., Sayers, J. R. and Grasby, J. A. (2007) Comparison of the catalytic parameters and reaction specificities of a phage and an archaeal flap endonuclease. J. Mol. Biol. 371, 34-48

5 Finger, L. D., Blanchard, S. M., Theimer, C. A., Sengerova, B., Singh, P., Chavez, V., Liu, F., Grasby, J. A. and Shen, B. (2009) The 3'-flap pocket of human flap endonuclease 1 is critical for substrate binding and catalysis. J. Biol. Chem. 284, 22184-22194

6 Kanai, Y., Ishikawa, G., Takeuchi, R., Ruike, T., Nakamura, R.-I., Ihara, A., Ohashi, T., Takata, K.-I., Kimura, S. and Sakaguchi, K. (2007) DmGEN shows a flap endonuclease activity, cleaving the blocked-flap structure and model replication fork. FEBs Journal 274, 3914-3927

7 Ip, S. C. Y., Rass, U., Blanco, M. G., Flynn, H. R., Skehel, J. M. and West, S. C. (2008) Identification of Holliday junction resolvases from humans and yeast. Nature 456, 357-361

8 West, S. C. (2009) The search for a human Holliday junction resolvase. Biochemical Society Transactions 37, 519-526

9 Lee, B.-I. and Wilson, D. M. (1999) The RAD2 Domain of Human Exonuclease 1 Exhibits 5' to 3'Exonuclease and Flap Structure-specific Endonuclease Activities. J. Biol. Chem. 274, 377763-37769

10 Hohl, M., Thorel, S. G., Clarkson, S. G. and Scharer, O. D. (2003) Strucutral Determinants for Substrate Binding and Catalysis by the structure specific endonuclease XPG. J. Biol. Chem. 278, 19500-19508

11 Kim, Y., Eom, S., Wang, J., Lee, D., Suh, S. and Steitz, T. (1995) Crystal structure of Thermus aquaticus DNA polymerase. Nature 376, 612-616 
12 Mueser, T. C., Nossal, N. G. and Hyde, C. C. (1996) Structure of bacteriophage T4 RNase H, a 5' to 3' RNA-DNA and DNA-DNA exonuclease with sequence similarity to the Rad2 family of eukaryotic proteins. Cell 85, 1101-1112

13 Ceska, T. A., Sayers, J. R., Stier, G. and Suck, D. (1996) A helical arch allowing single-stranded DNA to thread through T5 5'- exonuclease. Nature 382, 90-93

14 Hosfield, D. J., Mol, C. D., Shen, B. H. and Tainer, J. A. (1998) Structure of the DNA repair and replication endonuclease and exonuclease FEN-1: Coupling DNA and PCNA binding to FEN-1 activity. Cell 95, 135-146

15 Hwang, K. Y., Baek, K., Kim, H. Y. and Cho, Y. (1998) The crystal structure of flap endonuclease I from Methanococcus jannaschii. Nat. Struct. Biol. 5, 707-713

16 Sakurai, S., Kitano, K., Yamaguchi, H., Hamada, K., Okada, K., Fukuda, K., Uchida, M., Ohtsuka, E., Morioka, H. and Hakoshima, T. (2005) Structural basis for recruitment of human flap endonuclease 1 to PCNA. EMBO J. 24, 683-693

17 Matsui, E., Musti, K. V., Abe, J., Yamasaki, K., Matsui, I. and Harata, K. (2002) Molecular structure and novel DNA binding sites located in loops of flap endonuclease-1 from Pyrococcus horikoshii. J. Biol. Chem. 277, 37840-37847

18 Chapados, B. R., Hosfield, D. J., Han, S., Qiu, J. Z., Yelent, B., Shen, B. H. and Tainer, J. A. (2004) Structural basis for FEN-1 substrate specificity and PCNAmediated activation in DNA replication and repair. Cell 116, 39-50

19 Devos, J. M., Tomanicek, S. J., Jones, C. E., Nossal, N. G. and Mueser, T. J. (2007) Crystal structure of bacteriophage T4 5' nuclease in complex with a branched DNA reveals how FEN-1 family nucleases bind their substrates. J. Biol. Chem. 282, 31713-31724

20 Dervan, J. J., Feng, M., Patel, D., Grasby, J. A., Artymiuk, P. J., Ceska, T. A. and Sayers, J. R. (2002) Interactions of mutant and wild-type flap endonucleases with oligonucleotide substrates suggest an alternative model for DNA binding. Proc. Natl. Acad. Sci. USA 99, 8542-8547

21 Allawi, H. T., Kaiser, M. W., Onufriev, A. V., Ma, W. P., Brogaard, A. E., Case, D. A., Neri, B. P. and Lyamichev, V. I. (2003) Modelling of flap endonuclease interactions with DNA substrate. J. Mol. Biol. 328, 537-554

22 Qiu, J. Z., Liu, R., Chapados, B. R., Sherman, M., Tainer, J. A. and Shen, B. H. (2004) Interaction interface of human flap endonuclease-1 with its DNA substrates. J. Biol. Chem. 279, 24394-24402

23 Thayer, M., Ahern, H., Xing, D., Cunningham, R. P. and Tainer, J. A. (1995) Novel DNA binding motifs in the DNA-repair enzyme endonuclease III structure. EMBO J. 14, 4108-4120

24 Kaiser, M. W., Lyamicheva, N., Ma, W. P., Miller, C., Neri, B., Fors, L. and Lyamichev, V. I. (1999) A comparison of eubacterial and archaeal structurespecific 5'-exonucleases. J. Biol. Chem. 274, 21387-21394

25 Friedrich-Heineken, E. and Hubscher, U. (2004) The Fen1 extrahelical 3 '-flap pocket is conserved from archaea to human and regulates DNA substrate specificity. Nucl. Acids Res. 32, 2520-2528

26 Beese, L. S. and Steitz, T. A. (1991) Structural basis for the 3'-5' exonuclease activity of Escherichia coli DNA polymerase I - a two-metal-ion mechanism. EMBO J. 10, 25-33 
27 Zheng, L., Li, M., Shan, J. X., Krishnamoorthi, R. and Shen, B. H. (2002) Distinct roles of two $\mathrm{Mg}^{2+}$ binding sites in regulation of murine flap endonuclease-1 activities. Biochemistry 41, 10323-10331

28 Feng, M., Patel, D., Dervan, J. J., Ceska, T., Suck, D., Haq, I. and Sayers, J. R. (2004) Roles of divalent metal ions in flap endonuclease-substrate interactions. Nat. Struct. Mol. Biol. 11, 450-456

29 Tock, M. R., Frary, E., Sayers, J. R. and Grasby, J. A. (2003) Dynamic evidence for metal ion catalysis in the reaction mediated by a flap endonuclease. EMBO J. 22, 995-1004

30 Syson, K., Tomlinson, C., Chapados, B. R., Sayers, J. R., Tainer, J. A., Williams, N. H. and Grasby, J. A. (2008) Three metal ions participate in T5 flap endonuclease catalysed DNA hydrolysis. J. Biol. Chem. 283, 28741-28746

31 Garforth, S. J. and Sayers, J. R. (1997) Structure-specific DNA binding by bacteriophage T5 5'->3' exonuclease. Nucl. Acids Res. 25, 3801-3807

32 Murante, R. S., Rust, L. and Bambara, R. A. (1995) Calf 5' to 3' exo/endonuclease must slide from a $5^{\prime}$ end of the substrate to perform structure-specific cleavage. J. Biol. Chem. 270, 30377-30383

33 Sarker, A. H., Tsutakawa, S. E., Kostek, S., Ng, C., Shin, D. S., Peris, M., Campeau, E., Tainer, J. A., Nogales, E. and Cooper, P. K. (2005) Recognition of RNA Polymerase II and Transcription Bubbles by XPG, CSB, and TFIIH: Insights for transcrition-coupled repair and cockayne syndrome. Molecular Cell 20, 187-198

34 Staresincic, L., Fagbemi, A. F., Enzlin, J. H., Gourdin, A. M., Wijgers, N., Dunand-Sauthier, I., Giglia-Mari, G., Clarkson, S. G., Verme, W. and Schärer, O. D. (2009) Coordination of dual incision and repair synthesis in human nucleotide excision repair. EMBO J. 28, 1111-1120 
Figure Legends

Figure 1. FEN family substrates and structural architecture.

(a) FEN and related enzymes recognise and specifically cleave a wide range of structurally-related DNA substrates: DOUBLE-FLAP (5' and 3' overlapping flaps), FLAP (5' flap), EXO (exonuclease substrate), GEN (gap-endonuclease substrate), BUBBLE (unpaired region flanked by duplex DNA, proposed substrate for XPG), HJ (Holliday junction, proposed substrate for GEN-1).

(b) Schematic representation of FEN family members: Flap endonuclease (FEN), Xeroderm pigmentosum-G (XPG), Exonuclease-I (EXO-1), Holliday junction endonuclease (GEN-1). Each enzyme consists of conserved N- and C-domains (white boxes; required for nuclease activity) separated by a variable length region (grey) that may form a helical clamp or arch. FEN structures show that the $\mathrm{N}$ - and C-domains cross each other, forming the active site at the junction of the two domains, near the helical arch/clamp region.

(c) The structure of AfFEN (light grey) bound to DNA (beige) (1RXW) reveals a binding site (cyan residues) for the 3' flap (red). This binding mode implies a kinked DNA substrate conformation, which positions the 5 ' flap near the active site for catalysis. This helical clamp (green) and H3TH (blue) are positioned to interact with the downstream portion of the substrate. This region of the substrate is present in (d) the structure of T4FEN bound to pseudo-Y DNA substrate (2IHN; same colouring as (c)). Both the helical clamp/arch (partially disordered) and H3TH motifs are observed to bind the DNA.

Figure 2. The active sites of flap endonucleases. Comparison of active sites from T5FEN (a; 1UT5), T4FEN (b; 1TFR), hFEN (c; 1UL1:X), and MjFEN (d; 1A77) illustrating the positional conservation of active site carboxylates (black) and metal ions ( $1 \& 2$, grey). Phage and bacterial FENs have an additional carboxylate (T5 Asp201, T4 Asp197) in the active site. Although two metal ions are bound in each active site, their positions vary. Metal 1 is bound in a similar position is all structures, but the coordination site of metal 2 is variable. The spacing between metal 1 and metal 2 in the structures are (a) T5FEN $8 \AA$, (b) T4FEN $6.3 \AA$, (c) hFEN $3.4 \AA$,(d) MjFEN $5 \AA$. In the structures of the archaeal (d) 
and human (c) enzymes, the $\mathrm{N}$-terminus of the protein occupies a similar position to metal 2 in the phage enzymes $(a, b)$.

\section{Figure 3.}

T4FEN-DNA, kinetic studies of metal ion dependence of FEN reactions and active site metal comparisons suggest that a conformational change is required for catalysis.

(a) Close up of the active site of the T4FEN-DNA complex (2IHN) with metals (pink and green) superimposed according to a substrate-free structure of T4FEN (1TFR). In the absence of metal ions, DNA is stabilised by contacts with hydrophobic residues (orange). The 5'-nucleobase is coloured red (red base) and is base paired. In this configuration, the phosphate diester corresponding to the major site of FEN reaction (black arrow) is located in the duplex region of the substrate at a distance of $7 \AA$ from metal 1 .

(b) Suggested unpairing (red base) of the duplex DNA to allow contact between the scissile phosphate diester (black) and metal 1 (green) and produce a functional complex. 
Figure 1
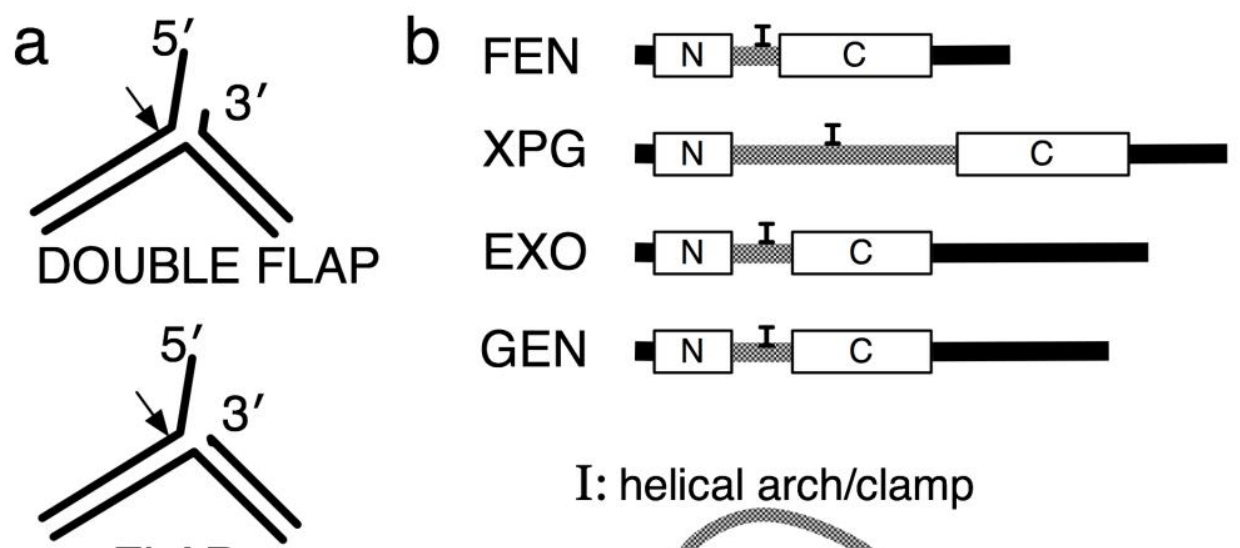

EXO $\mathrm{N}^{\mathrm{I}} \mathrm{C}$

FLAP
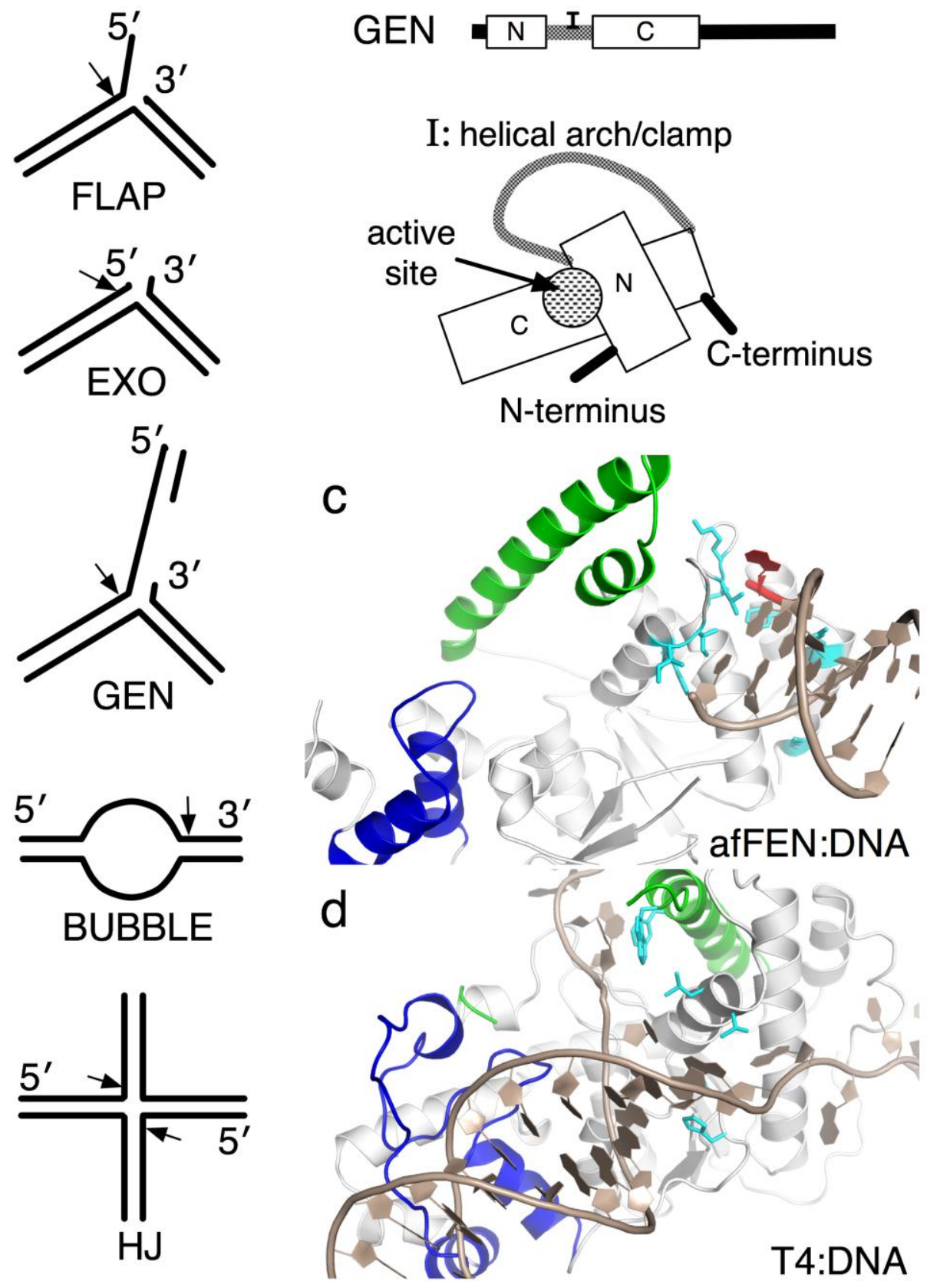
Figure 2

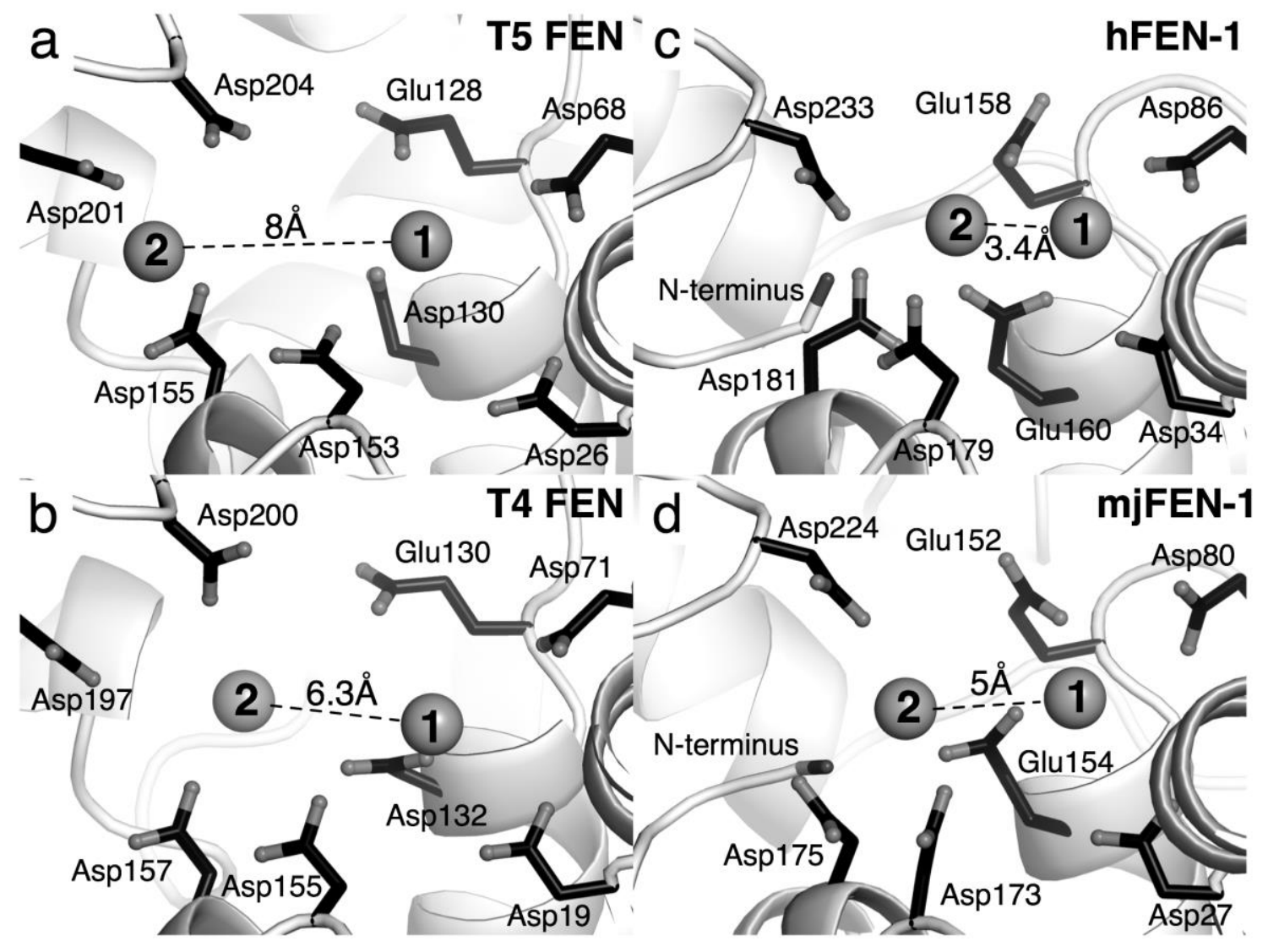

Figure 3

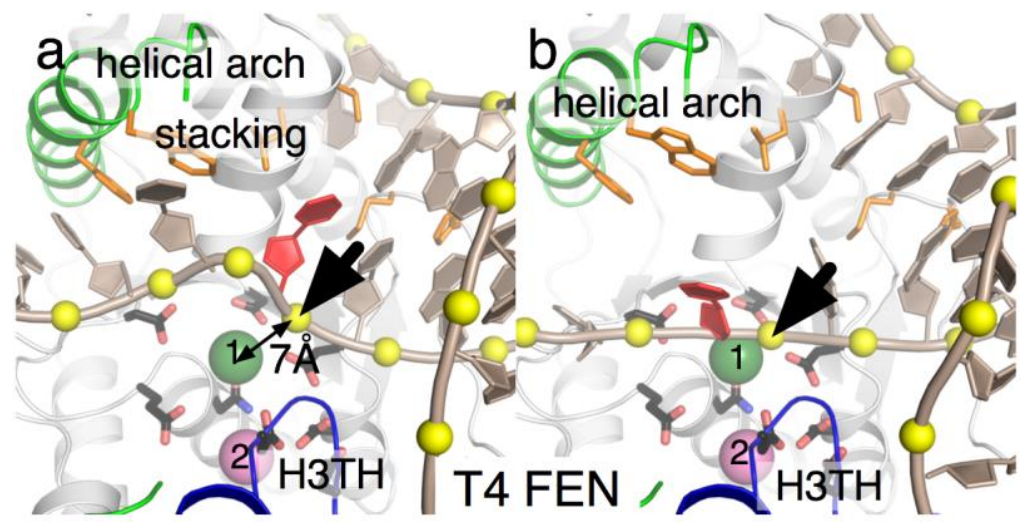

